\title{
Certain Geometric Properties of Normalized Wright Functions
}

\author{
Mohsan Raza, ${ }^{1}$ Muhey U Din, ${ }^{1}$ and Sarfraz Nawaz Malik ${ }^{2}$ \\ ${ }^{1}$ Department of Mathematics, Government College University Faisalabad, Faisalabad, Pakistan \\ ${ }^{2}$ Department of Mathematics, COMSATS Institute of Information Technology, Wah Cantt, Pakistan
}

Correspondence should be addressed to Sarfraz Nawaz Malik; snmalik110@yahoo.com

Received 15 October 2016; Accepted 4 December 2016

Academic Editor: Adrian Petrusel

Copyright (C) 2016 Mohsan Raza et al. This is an open access article distributed under the Creative Commons Attribution License, which permits unrestricted use, distribution, and reproduction in any medium, provided the original work is properly cited.

In this article, we find some geometric properties like starlikeness, convexity of order $\alpha$, close-to-convexity of order $(1+\alpha) / 2$, and close-to-convexity of normalized Wright functions with respect to the certain functions. The sufficient conditions for the normalized Wright functions belonging to the classes $\mathscr{T}_{\gamma}(\alpha)$ and $\mathscr{L}_{\gamma}(\alpha)$ are the part of our investigations. We also obtain the conditions on normalized Wright function to belong to the Hardy space $\mathscr{H}^{p}$.

\section{Introduction and Preliminaries}

Let $\mathscr{H}$ denote the class of all analytic functions in the open unit disk $\mathscr{U}=\{z:|z|<1\}$ and $\mathscr{H}^{\infty}$ denote the space of all bounded functions on $\mathscr{H}$. This is Banach algebra with respect to the norm

$$
\|f\|_{\infty}=\sup _{z \in \mathscr{U}}|f(z)|
$$

We denote $\mathscr{H}^{p}, 0<p<\infty$, for the space of all functions $f \in \mathscr{H}$ such that $|f|^{p}$ admits a harmonic majorant. $\mathscr{H}^{p}$ is a Banach space if the norm of $f$ is defined to be $p$ th root of the least harmonic majorant of $|f|^{p}$ for some fixed $z \in \mathcal{U}$. Another equivalent definition of norm is given as follows: let $f \in \mathscr{H}$, and set

$$
\begin{aligned}
& M_{p}(r, f) \\
& \quad= \begin{cases}\left(\frac{1}{2 \pi} \int_{0}^{2 \pi}\left|f\left(r e^{i \theta}\right)\right|^{p} d \theta\right)^{1 / p}, & 0<p<\infty, \\
\max \{|f(z)|:|z| \leq r\}, & p=\infty .\end{cases}
\end{aligned}
$$

Then the function $f \in \mathscr{H}^{p}$ if $M_{p}(r, f)$ is bounded for all $r \in$ $[0,1)$. It is clear that

$$
\mathscr{H}^{\infty} \subset \mathscr{H}^{q} \subset \mathscr{H}^{p}, \quad 0<q<p<\infty .
$$

For some details, see [1]. It is also known [1] that $\operatorname{Re}\left\{f^{\prime}(z)\right\}>$ 0 in $\mathcal{U}$, and then

$$
\begin{aligned}
& f^{\prime} \in \mathscr{H}^{q}, \quad q<1, \\
& f \in \mathscr{H}^{q /(1-q)}, \quad 0<q<1 .
\end{aligned}
$$

Let $\mathscr{A}$ be the class of functions $f$ of the form

$$
f(z)=z+\sum_{n=2}^{\infty} a_{n} z^{n}
$$

analytic in the open unit disc $\mathcal{U}=\{z:|z|<1\}$, and $\mathcal{S}$ denote the class of all functions in $\mathscr{A}$ which are univalent in $\mathscr{U}$. Let $\mathcal{S}^{*}(\alpha), \mathscr{C}(\alpha)$, and $\mathscr{K}(\alpha)$ denote the classes of starlike, convex, and close-to-convex functions of order $\alpha$, respectively, and they are defined as

$$
\begin{aligned}
& \mathcal{S}^{*}(\alpha)=\left\{f: f \in \mathscr{A}, \operatorname{Re}\left(\frac{z f^{\prime}(z)}{f(z)}\right)>\alpha, z \in \mathcal{U}, \alpha\right. \\
& \in[0,1)\}, \\
& \mathscr{C}(\alpha)=\left\{f: f \in \mathscr{A}, \operatorname{Re}\left(1+\frac{z f^{\prime \prime}(z)}{f^{\prime}(z)}\right)>\alpha, z\right. \\
& \in \mathscr{U}, \alpha \in[0,1)\},
\end{aligned}
$$




$$
\begin{aligned}
& \mathscr{K}(\alpha)=\left\{f: f \in \mathscr{A}, \operatorname{Re}\left(\frac{z f^{\prime}(z)}{g(z)}\right)>\alpha, z \in \mathcal{U}, \alpha\right. \\
& \left.\quad \in[0,1), g \in \mathcal{S}^{*}\right\} .
\end{aligned}
$$

It is clear that

$$
\begin{gathered}
\mathcal{S}^{*}(0)=\mathcal{S}^{*}, \\
\mathscr{C}(0)=\mathscr{C}, \\
\mathscr{K}(0)=\mathscr{K}
\end{gathered}
$$

are the classes of starlike, convex, and close-to-convex functions, respectively. Also consider the subclasses $\mathscr{T}_{\gamma}(\alpha)$ and $\mathscr{L}_{\gamma}(\alpha)$ of $\mathscr{A}$, defined by the following relations:

$$
\begin{aligned}
& \mathscr{T}_{\gamma}(\alpha)=\left\{f \in \mathscr{A}: \operatorname{Re}\left(\frac{z f^{\prime}(z)+\gamma z^{2} f^{\prime \prime}(z)}{(1-\gamma) f(z)+\gamma z f^{\prime}(z)}\right)\right. \\
& >\alpha\}, \\
& \mathscr{L}_{\gamma}(\alpha)=\{f \in \mathscr{A}: \\
& \operatorname{Re}\left(\frac{\gamma z^{3} f^{\prime \prime \prime}(z)+(1+2 \gamma) z^{2} f^{\prime \prime}(z)+z f^{\prime}(z)}{z f^{\prime}(z)+\gamma z^{2} f^{\prime \prime}(z)}\right) \\
& >\alpha\},
\end{aligned}
$$

where $0 \leq \alpha, \gamma<1$. The purpose of these subclasses is that when we put $\gamma=0$ in (8), we get $\mathscr{T}_{0}(\alpha)=\mathcal{S}^{*}(\alpha)$ and $\mathscr{L}_{0}(\alpha)=\mathscr{C}(\alpha)$. The sufficient coefficient conditions by which a function $f \in \mathscr{A}$ as defined in (5) belongs to the classes $\mathscr{T}_{\gamma}(\alpha)$ and $\mathscr{L}_{\gamma}(\alpha)$ are

$$
\begin{gathered}
\sum_{n=2}^{\infty}(n \gamma-\gamma+1)(n-\alpha)\left|a_{n}\right| \leq 1-\alpha, \\
\sum_{n=2}^{\infty} n(n \gamma-\gamma+1)(n-\alpha)\left|a_{n}\right| \leq 1-\alpha,
\end{gathered}
$$

respectively. For some details about these classes, see [2, 3]. Recently, Baricz [4] introduced the classes

$$
\begin{aligned}
& \mathscr{P}_{\eta}(\alpha)=\left\{p: p \in \mathscr{H}, p(0)=1, \operatorname{Re}\left(e^{i \eta}(p(z)-\alpha)\right)\right. \\
& >0, z \in \mathcal{U}, \alpha \in[0,1), \eta \in \mathbb{R}\}, \\
& \mathscr{R}_{\eta}(\alpha)=\left\{f: f \in \mathscr{A}, \operatorname{Re}\left(e^{i \eta}\left(f^{\prime}(z)-\alpha\right)\right)>0, z\right. \\
& \quad \in \mathscr{U}, \alpha \in[0,1), \eta \in \mathbb{R}\} .
\end{aligned}
$$

For $\eta=0$, we denote the classes $\mathscr{P}_{0}(\alpha)$ and $\mathscr{R}_{0}(\alpha)$ by $\mathscr{P}(\alpha)$ and $\mathscr{R}(\alpha)$, respectively. Also for $\eta=0$ and $\alpha=0$, we have the classes $\mathscr{P}$ and $\mathscr{R}$.
Let $f \in \mathscr{A}$ given by (5) and $g \in \mathscr{A}$ given by

$$
g(z)=z+\sum_{n=2}^{\infty} b_{n} z^{n}
$$

and then Hadamard product (or convolution) of $f$ and $g$ is defined as

$$
(f * g)(z)=z+\sum_{n=2}^{\infty} a_{n} b_{n} z^{n} \quad(z \in \mathcal{U}) .
$$

Recently, Prajapat [5] studied some geometric properties of Wright function

$$
W_{\lambda, \mu}(z)=\sum_{n=0}^{\infty} \frac{z^{n}}{n ! \Gamma(\lambda n+\mu)}, \quad \lambda>-1, \mu \in \mathbb{C} .
$$

This series is absolutely convergent in $\mathbb{C}$ for $\lambda>-1$ and absolutely convergent in open unit disc $\mathcal{U}$ for $\lambda=-1$. Furthermore this function is entire. The Wright functions were introduced by Wright [6] and have been used in the asymptotic theory of partitions, in the theory of integral transforms of the Hankel type and in Mikusinski operational calculus. Recently, Wright functions have been found in the solution of partial differential equations of fractional order. It was found that the corresponding Green functions can be represented in terms of the Wright function $[7,8]$. For positive rational number $\lambda$, the Wright function can be represented in terms of generalized hypergeometric function. For some details, see [9, Section 2.1]. In particular, the function $W_{1, v+1}\left(-z^{2} / 4\right)$ can be expressed in terms of the Bessel functions $J_{v}$, given as

$$
J_{v}(z)=\left(\frac{z}{2}\right)^{2} W_{1, v+1}\left(-\frac{z^{2}}{4}\right)=\sum_{n=0}^{\infty} \frac{(-1)^{n}(z / 2)^{2 n+v}}{n ! \Gamma(n+v+1)} .
$$

The Wright function generalizes various functions like Array function, Whittaker function, entire auxiliary functions, and so forth. For more details, we refer to [9]. Prajapat [5] discussed some geometric properties of the Wright functions,

$$
\mathrm{W}_{\lambda, \mu}(z)=\sum_{n=0}^{\infty} \frac{\Gamma(\mu)}{n ! \Gamma(\lambda n+\mu)} z^{n}
$$

$$
\lambda>-1, \mu>0, z \in \mathcal{U}
$$

and their normalization of the form

$$
\begin{array}{r}
z \mathrm{~W}_{\lambda, \mu}(z)=\mathscr{W}_{\lambda, \mu}(z)=z+\sum_{n=1}^{\infty} \frac{\Gamma(\mu)}{n ! \Gamma(\lambda n+\mu)} z^{n+1}, \\
\lambda>-1, \mu>0, \quad z \in \mathcal{U},
\end{array}
$$

where $\lambda+\mu>0$. The Pochhammer (or Appell) symbol, defined in terms of Euler's gamma functions, is given as $(x)_{n}=\Gamma(x+n) / \Gamma(x)=x(x+1) \cdots(x+n-1)$. We refer for some geometric properties of special functions like hypergeometric functions [10, 11], Bessel functions [4, 12-14], and Struve functions $[15,16]$.

We need the following results to prove our results. 
Lemma 1 (see [17]). If $f \in \mathscr{A}$ satisfies the inequality

$$
\left|z f^{\prime \prime}(z)\right|<\frac{1-\alpha}{4}, \quad(z \in \mathscr{U}, 0 \leq \alpha<1)
$$

then

$$
\operatorname{Re} f^{\prime}(z)>\frac{1+\alpha}{4}, \quad(z \in \mathcal{U}, 0 \leq \alpha<1) .
$$

Lemma 2 (see [18]). If the function $f(z)=z+a_{2} z^{2}+\cdots+a_{n} z^{n}+$ $\cdots$ is analytic in $\mathcal{U}$ and in addition $1 \geq 2 a_{2} \geq \cdots \geq n a_{n} \geq \cdots \geq$ 0 or $1 \leq 2 a_{2} \leq \cdots \leq n a_{n} \leq \cdots \leq 2$, then $f(z)$ is close-to-convex function with respect to the convex function $z \rightarrow-\log (1-z)$. Moreover, if the odd function $g(z)=z+b_{3} z^{3}+\cdots+b_{2 n-1} z^{2 n-1}+$ $\cdots$ is analytic in $\mathcal{U}$ and if $1 \geq 3 b_{3} \geq \cdots \geq(2 n+1) b_{2 n+1} \geq \cdots \geq$ 0 or $1 \leq 3 b_{3} \leq \cdots \leq(2 n+1) b_{2 n+1} \leq \cdots \leq 2$, then $g(z)$ is univalent in $\mathcal{U}$.

Lemma 3 (see [19]). $\mathscr{P}_{0}(\alpha) * \mathscr{P}_{0}(\beta) \subset \mathscr{P}_{0}(\gamma)$, where $\gamma=$ $1-2(1-\alpha)(1-\beta)$ with $\alpha, \beta<1$ and the value of $\gamma$ is the best possible.

Lemma 4 (see [20]). For $\alpha, \beta<1$ and $\gamma=1-2(1-\alpha)(1-$ $\beta)$, we have $\mathscr{R}_{0}(\alpha) * \mathscr{R}_{0}(\beta) \subset \mathscr{R}_{0}(\gamma)$ or equivalently $\mathscr{P}(\alpha) *$ $\mathscr{P}_{0}(\beta) \subset \mathscr{P}_{0}(\gamma)$

Lemma 5 (see [21]). If the function $f$, convex of order $\alpha$, where $\alpha \in[0,1)$, is not of the form

$$
f(z)=\left\{\begin{array}{lr}
k+d z\left(1-z e^{i \gamma}\right)^{2 \alpha-1}, & \alpha \neq \frac{1}{2}, \\
k+d \log \left(1-z e^{i \gamma}\right), & \alpha=\frac{1}{2}
\end{array}\right.
$$

for some complex numbers $k$ and $d$ and for some real number $\gamma$, then the following statements hold:

(i) There exists $\delta=\delta(f)>0$ such that $f^{\prime} \in \mathscr{H}^{\delta+1 /[2(1-\alpha)]}$.

(ii) If $\alpha \in[0,1 / 2)$, then there exists $\tau=\tau(f)>0$ such that $f \in \mathscr{H}^{\tau+1 /(1-2 \alpha)}$.

(iii) If $\alpha \geq 1 / 2$, then $f \in \mathscr{H}^{\infty}$.

\section{Main Results}

Theorem 6. Let $\lambda, \mu \in \mathbb{R}$ with $\alpha \in[0,1)$ and $z \in \mathcal{U}$. Then the following assertions are true:

(i) If $\lambda \geq 1$ and $\mu>\left((2-\alpha)+\sqrt{5 \alpha^{2}-16 \alpha+12}\right) / 2(1-\alpha)$, then $\mathscr{W}_{\lambda, \mu} \in \mathcal{S}^{*}(\alpha)$.

(ii) If $\lambda \geq 1$ and $\mu>\left((4-\alpha)+\sqrt{5 \alpha^{2}-28 \alpha+32}\right) / 2(1-\alpha)$, then $\mathscr{W}_{\lambda, \mu} \in \mathscr{C}(\alpha)$.

(iii) If $\lambda \geq 1$ and $\mu>(6+\sqrt{48-12 \alpha}) /(1-\alpha)$, then $\mathscr{W}_{\lambda, \mu} \in$ $\mathscr{K}((1+\alpha) / 2)$.

(iv) If $\lambda \geq 1$ and $\mu>(1+\sqrt{5-4 \alpha}) / 2(1-\alpha)$, then $\mathscr{W}_{\lambda, \mu} / z \epsilon$ $\mathscr{P}(\alpha)$.
Proof. (i) To prove that $\mathscr{W}_{\lambda, \mu} \in \mathcal{S}^{*}(\alpha)$, we have to show that $\left|z \mathscr{W}_{\lambda, \mu}^{\prime}(z) / \mathscr{W}_{\lambda, \mu}(z)-1\right|<1-\alpha$. By using the well-known triangle inequality

$$
\left|z_{1}+z_{2}\right| \leq\left|z_{1}\right|+\left|z_{2}\right|
$$

with the inequality $\Gamma(\mu+n) \leq \Gamma(\mu+n \lambda), n \in \mathbb{N}$, which is equivalent to $\Gamma(\mu) / \Gamma(\lambda n+\mu) \leq 1 /(\mu(\mu+1) \cdots(\mu+n-1))=$ $1 /(\mu)_{n}, n \in \mathbb{N}$, and the inequality

$$
n !(\mu+1)_{n-1} \geq n(\mu+1)^{n-1}, \quad n \in \mathbb{N},
$$

we obtain

$$
\begin{aligned}
& \left|\mathscr{W}_{\lambda, \mu}^{\prime}(z)-\frac{\mathscr{W}_{\lambda, \mu}(z)}{z}\right| \leq \sum_{n=1}^{\infty} \frac{n \Gamma(\mu)}{n ! \Gamma(\lambda n+\mu)} \\
& \quad \leq \sum_{n=1}^{\infty} \frac{n \Gamma(\mu)}{n ! \Gamma(n+\mu)} \quad(\lambda \geq 1) \\
& \quad \leq \frac{1}{\mu} \sum_{n=1}^{\infty}\left(\frac{1}{\mu+1}\right)^{n-1}=\frac{\mu+1}{\mu^{2}}, \quad \mu>0, z \in \mathcal{U} .
\end{aligned}
$$

Also consider

$$
\frac{\mathscr{W}_{\lambda, \mu}(z)}{z}=1+\sum_{n=1}^{\infty} \frac{\Gamma(\mu)}{n ! \Gamma(\lambda n+\mu)} z^{n} .
$$

Since $\Gamma(n+\mu) \leq \Gamma(\lambda n+\mu)$, therefore by using the reverse triangle inequality

$$
|| z_{1}|-| z_{2}|| \leq\left|z_{1}+z_{2}\right|
$$

and the inequality, $n !(\mu+1)_{n-1} \geq(\mu+1)^{n-1}, n \in \mathbb{N}$, we get

$$
\begin{aligned}
\left|\frac{\mathscr{W}_{\lambda, \mu}(z)}{z}\right| & =\left|1+\sum_{n=1}^{\infty} \frac{\Gamma(\mu)}{n ! \Gamma(\lambda n+\mu)} z^{n}\right| \\
& \geq 1-\sum_{n=1}^{\infty} \frac{1}{n !(\mu)_{n}} \geq 1-\frac{1}{\mu} \sum_{n=1}^{\infty}\left(\frac{1}{\mu+1}\right)^{n-1} \\
& =\frac{\mu^{2}-\mu-1}{\mu^{2}}, \quad(\mu>0) .
\end{aligned}
$$

By combining (23) and (26), we get

$$
\left|\frac{z \mathscr{W}_{\lambda, \mu}^{\prime}(z)}{\mathscr{W}_{\lambda, \mu}(z)}-1\right| \leq \frac{\mu+1}{\mu^{2}-\mu-1}<1-\alpha .
$$

So $\mathscr{W}_{\lambda, \mu}(z)$ is starlike function of order $\alpha$, where $0 \leq \alpha<$ $1-(\mu+1) /\left(\mu^{2}-\mu-1\right)$.

(ii) To prove that $\mathscr{W}_{\lambda, \mu} \in \mathscr{C}(\alpha)$, we have to show that $\left|z \mathscr{W}_{\lambda, \mu}^{\prime \prime}(z) / \mathscr{W}_{\lambda, \mu}^{\prime}(z)\right|<1-\alpha$. By using the well-known triangle inequality

$$
\left|z_{1}+z_{2}\right| \leq\left|z_{1}\right|+\left|z_{2}\right|
$$


with the inequality $\Gamma(\mu+n) \leq \Gamma(\mu+n \lambda), n \in \mathbb{N}$, which is equivalent to $\Gamma(\mu) / \Gamma(\lambda n+\mu) \leq 1 /(\mu(\mu+1) \cdots(\mu+n-1))=$ $1 /(\mu)_{n}, n \in \mathbb{N}$, and the inequalities

$$
\begin{gathered}
n ! \geq \frac{n(n+1)}{2^{n}}, \\
(\mu+1)_{n-1} \geq(\mu+1)^{n-1}, \quad \\
n \in \mathbb{N},
\end{gathered}
$$

we have

$$
\begin{aligned}
&\left|z \mathscr{W}_{\lambda, \mu}^{\prime \prime}(z)\right|=\left|\sum_{n=1}^{\infty} \frac{\Gamma(\mu) n(n+1)}{n ! \Gamma(\lambda n+\mu)} z^{n}\right| \\
& \leq \sum_{n=1}^{\infty} \frac{n(n+1) 2^{n} \Gamma(\mu)}{n(n+1) \Gamma(\lambda n+\mu)} \\
& \leq \frac{2}{\mu} \sum_{n=1}^{\infty}\left(\frac{2}{\mu+1}\right)^{n-1}=\frac{2(\mu+1)}{\mu(\mu-1)}, \\
&(\mu>1) .
\end{aligned}
$$

Since $\Gamma(n+\mu) \leq \Gamma(\lambda n+\mu)$, therefore by using the reverse triangle inequality

$$
|| z_{1}|-| z_{2}|| \leq\left|z_{1}+z_{2}\right|
$$

and the inequality, $n !(\mu+1)_{n-1} \geq n(\mu+1)^{n-1}, n \in \mathbb{N}$, we get

$$
\begin{aligned}
&\left|\mathscr{W}_{\lambda, \mu}^{\prime}(z)\right| \geq 1-\sum_{n=1}^{\infty} \frac{n \Gamma(\mu)}{n ! \Gamma(n+\mu)} \\
&=1-\frac{1}{\mu} \sum_{n=1}^{\infty} \frac{n}{n !(\mu+1)_{n-1}} \\
& \geq 1-\frac{1}{\mu} \sum_{n=1}^{\infty}\left(\frac{1}{\mu+1}\right)^{n-1}=\frac{\mu^{2}-\mu-1}{\mu^{2}}, \\
& \quad(\mu>0) .
\end{aligned}
$$

Combining (30) and (32), we have

$$
\left|\frac{z \mathscr{W}_{\lambda, \mu}^{\prime \prime}(z)}{\mathscr{W}_{\lambda, \mu}^{\prime}(z)}\right| \leq \frac{3(\mu+1)}{\mu^{2}-\mu-1}<1-\alpha .
$$

This implies that $\mathscr{W}_{\lambda, \mu}$ is convex function of order $\alpha$, where $0 \leq \alpha<1-3(\mu+1) /\left(\mu^{2}-\mu-1\right)$.

(iii) Using inequality (30) and Lemma 1, we have

$$
\left|z \mathscr{W}_{\lambda, \mu}^{\prime \prime}(z)\right| \leq \frac{3(\mu+1)}{\mu^{2}} \leq \frac{1-\alpha}{4},
$$

where $0 \leq \alpha<1-12(\mu+1) / \mu^{2}$ and $\mu>(6+$ $\sqrt{48-12 \alpha}) /(1-\alpha)$. This shows that $\mathscr{W}_{\lambda, \mu} \in \mathscr{K}((1+\alpha) / 2)$. Therefore, $\operatorname{Re} \mathscr{W}_{\lambda, \mu}^{\prime}(z)>(1+\alpha) / 2$. (iv) To prove that $\mathscr{W}_{\lambda, \mu} / z \in \mathscr{P}(\alpha)$, we have to show that $|g(z)-1|<1$, where $g(z)=\left(\mathscr{W}_{\lambda, \mu}(z) / z-\alpha\right) /(1-\alpha)$. By using the well-known triangle inequality

$$
\left|z_{1}+z_{2}\right| \leq\left|z_{1}\right|+\left|z_{2}\right|
$$

with the inequality $\Gamma(\mu+n) \leq \Gamma(\mu+n \lambda), n \in \mathbb{N}$, and the inequality

$$
n !(\mu+1)_{n-1} \geq(\mu+1)^{n-1}, \quad n \in \mathbb{N},
$$

we have

$$
\begin{aligned}
|g(z)-1| & =\left|\frac{1}{1-\alpha} \sum_{n=1}^{\infty} \frac{\Gamma(\mu)}{n ! \Gamma(\lambda n+\mu)} z^{n}\right| \\
& \leq \frac{1}{1-\alpha} \frac{1}{\mu} \sum_{n=1}^{\infty} \frac{1}{n !(\mu+1)_{n-1}} \\
& \leq \frac{1}{1-\alpha} \frac{1}{\mu} \sum_{n=1}^{\infty}\left(\frac{1}{\mu+1}\right)^{n-1}=\frac{\mu+1}{\mu^{2}(1-\alpha)} .
\end{aligned}
$$

Therefore, $\mathscr{W}_{\lambda, \mu} / z \in \mathscr{P}(\alpha)$ for $0<\alpha<1-(\mu+1) / \mu^{2}$.

Putting $\alpha=0$ in Theorem 6, we have the following result.

Corollary 7. Let $\lambda, \mu \in \mathbb{R}$ and $z \in \mathcal{U}$. Then the following assertions are true:

(i) If $\lambda \geq 1$ and, $\mu>1+\sqrt{3}$, then $\mathscr{W}_{\lambda, \mu} \in \mathcal{S}^{*}$.

(ii) If $\lambda \geq 1$ and $\mu>2+2 \sqrt{2}$, then $\mathscr{W}_{\lambda, \mu} \in \mathscr{C}$.

(iii) If $\lambda \geq 1$ and $\mu>6+4 \sqrt{3}$, then $\mathscr{W}_{\lambda, \mu} \in \mathscr{K}(1 / 2)$.

(iv) If $\lambda \geq 1$ and $\mu>(1+\sqrt{5}) / 2$, then $\mathscr{W}_{\lambda, \mu} / z \in \mathscr{P}$.

Theorem 8. If $\alpha \in[0,1), \mu \geq 0$, and $\lambda \geq-1$, then a sufficient condition for $z \mathrm{~W}_{\lambda, \mu}$ to be in $\mathscr{T}_{\gamma}(\alpha)$ is

$$
\frac{\gamma \mathrm{W}_{\lambda, \mu}^{\prime \prime}(1)}{1-\alpha}+\frac{(1-\alpha \gamma+2 \gamma)}{1-\alpha} \mathrm{W}_{\lambda, \mu}^{\prime}(1)+\mathrm{W}_{\lambda, \mu}(1) \leq 2 .
$$

Proof. Consider the identity,

$$
z \mathrm{~W}_{\lambda, \mu}(z)=z+\sum_{n=2}^{\infty} b_{n-1} z^{n}
$$

By using (9), we will only show that

$$
\sum_{n=2}^{\infty}(n \gamma-\gamma+1)(n-\alpha) b_{n-1}(\lambda, \mu) \leq 1-\alpha,
$$

where $b_{n-1}(\lambda, \mu)=\Gamma(\mu) / n ! \Gamma(\lambda n+\mu)$. 
Now,

$$
\begin{aligned}
& \sum_{n=2}^{\infty}(n \gamma-\gamma+1)(n-\alpha) b_{n-1}(\lambda, \mu) \\
& =\gamma \sum_{n=2}^{\infty} n^{2} b_{n-1}(\lambda, \mu) \\
& +(1-\gamma(1+\alpha)) \sum_{n=2}^{\infty} n b_{n-1}(\lambda, \mu) \\
& +\alpha(\gamma-1) \sum_{n=2}^{\infty} b_{n-1}(\lambda, \mu) .
\end{aligned}
$$

From (39), a little simplification yields

$$
z^{2} \mathrm{~W}_{\lambda, \mu}^{\prime}(z)+z \mathrm{~W}_{\lambda, \mu}(z)=z+\sum_{n=2}^{\infty} n b_{n-1}(\lambda, \mu) z^{n} .
$$

Differentiating (42) two times with respect to $z$, we have

$$
\begin{aligned}
& z^{3} \mathrm{~W}_{\lambda, \mu}^{\prime \prime}(z)+3 z^{2} \mathrm{~W}_{\lambda, \mu}^{\prime}(z)+z \mathrm{~W}_{\lambda, \mu}(z) \\
& =z+\sum_{n=2}^{\infty} n^{2} b_{n-1}(\lambda, \mu) z^{n} \\
& z^{4} \mathrm{~W}_{\lambda, \mu}^{\prime \prime \prime \prime}+6 z^{3} \mathrm{~W}_{\lambda, \mu}^{\prime \prime}(z)+7 z^{2} \mathrm{~W}_{\lambda, \mu}^{\prime}(z)+z \mathrm{~W}_{\lambda, \mu}(z) \\
& =z+\sum_{n=2}^{\infty} n^{3} b_{n-1}(\lambda, \mu) z^{n} .
\end{aligned}
$$

Now for $|z|=1$, the expressions (39), (42), and (43) become

$$
\begin{aligned}
& \mathrm{W}_{\lambda, \mu}(1)=1+\sum_{n=2}^{\infty} b_{n-1}, \\
& \mathrm{~W}_{\lambda, \mu}^{\prime}(1)+\mathrm{W}_{\lambda, \mu}(1)=1+\sum_{n=2}^{\infty} n b_{n-1}(\lambda, \mu), \\
& \mathrm{W}_{\lambda, \mu}^{\prime \prime}(1)+3 \mathrm{~W}_{\lambda, \mu}^{\prime}(1)+\mathrm{W}_{\lambda, \mu}(1) \\
& =1+\sum_{n=2}^{\infty} n^{2} b_{n-1}(\lambda, \mu) .
\end{aligned}
$$

By using the above expressions, (41) becomes

$$
\begin{aligned}
& \sum_{n=2}^{\infty}(n \gamma-\gamma+1)(n-\alpha) b_{n-1}(\lambda, \mu) \\
&= \gamma \mathrm{W}_{\lambda, \mu}^{\prime \prime}(1)+(1-\alpha \gamma+2 \gamma) \mathrm{W}_{\lambda, \mu}^{\prime}(1) \\
&+(1-\alpha)\left(\mathrm{W}_{\lambda, \mu}(1)-1\right),
\end{aligned}
$$

And it is bounded above by $1-\alpha$ if (38) holds. Thus the proof is completed.

Corollary 9. The normalized Wright function is starlike of order $0 \leq \alpha<1$ with respect to the origin if

$$
\frac{\mathrm{W}_{\lambda, \mu}^{\prime}(1)}{1-\alpha}+\mathrm{W}_{\lambda, \mu}(1) \leq 2 \text {. }
$$

Theorem 10. If $\alpha \in[0,1), \mu \geq 0$, and $\lambda \geq-1$, then a sufficient condition for $z \mathrm{~W}_{\lambda, \mu}$ to be in $\mathscr{L}_{\gamma}(\alpha)$ is

$$
\begin{aligned}
\gamma \mathrm{W}_{\lambda, \mu}^{\prime \prime \prime}(1)+\frac{(5 \gamma+1-\alpha \gamma) \mathrm{W}_{\lambda, \mu}^{\prime \prime}(1)}{1-\alpha} & \\
& +\frac{(4 \gamma-2 \alpha \gamma-\alpha+3)}{1-\alpha} \mathrm{W}_{\lambda, \mu}^{\prime}(1)+\mathrm{W}_{\lambda, \mu}(1) \leq 2 .
\end{aligned}
$$

Proof. Consider the identity

$$
z \mathrm{~W}_{\lambda, \mu}(z)=z+\sum_{n=2}^{\infty} b_{n-1} z^{n}
$$

By using the (10), we will only show that

$$
\sum_{n=2}^{\infty} n(n \gamma-\gamma+1)(n-\alpha) b_{n-1}(\lambda, \mu) \leq 1-\alpha
$$

where $b_{n-1}(\lambda, \mu)=\Gamma(\mu) / n ! \Gamma(\lambda n+\mu)$.

Now,

$$
\begin{aligned}
& \sum_{n=2}^{\infty} n(n \gamma-\gamma+1)(n-\alpha) b_{n-1}(\lambda, \mu) \\
& =\gamma \sum_{n=2}^{\infty} n^{3} b_{n-1}(\lambda, \mu) \\
& \quad+(1-\gamma(1+\alpha)) \sum_{n=2}^{\infty} n^{2} b_{n-1}(\lambda, \mu) \\
& \quad+\alpha(\gamma-1) \sum_{n=2}^{\infty} n b_{n-1}(\lambda, \mu)
\end{aligned}
$$

Now for $|z|=1$ and using (42), (43), and (44), expression (51) becomes

$$
\begin{aligned}
& \sum_{n=2}^{\infty} n(n \gamma-\gamma+1)(n-\alpha) b_{n-1}(\lambda, \mu)=\gamma\left(\mathrm{W}_{\lambda, \mu}^{\prime \prime \prime}(1)\right. \\
& \left.\quad+7 \mathrm{~W}_{\lambda, \mu}^{\prime \prime}(1)+6 \mathrm{~W}_{\lambda, \mu}^{\prime}(1)+\mathrm{W}_{\lambda, \mu}(1)-1\right)+(1 \\
& \quad-\gamma(1+\alpha))\left(\mathrm{W}_{\lambda, \mu}^{\prime \prime}(1)+3 \mathrm{~W}_{\lambda, \mu}^{\prime}(1)+\mathrm{W}_{\lambda, \mu}(1)-1\right) \\
& \quad+\alpha(\gamma-1)\left(\mathrm{W}_{\lambda, \mu}^{\prime}(1)+\mathrm{W}_{\lambda, \mu}(1)-1\right)=\gamma \mathrm{W}_{\lambda, \mu}^{\prime \prime \prime}(1) \\
& \quad+(5 \gamma+1-\alpha \gamma) \mathrm{W}_{\lambda, \mu}^{\prime \prime}(1)+(4 \gamma-2 \alpha \gamma-\alpha+3) \\
& \quad \cdot \mathrm{W}_{\lambda, \mu}^{\prime}(1)+(1-\alpha)\left(\mathrm{W}_{\lambda, \mu}(1)-1\right)
\end{aligned}
$$

and is bounded above by $1-\alpha$ if (48) holds, which is the required result.

Corollary 11. The normalized Wright function is convex of order $\alpha, 0 \leq \alpha<1$, with respect to the origin if

$$
\frac{\mathrm{W}_{\lambda, \mu}^{\prime \prime}(1)}{1-\alpha}+\frac{(3-\alpha)}{1-\alpha} \mathrm{W}_{\lambda, \mu}^{\prime}(1)+\mathrm{W}_{\lambda, \mu}(1) \leq 2 .
$$




\section{Close-to-Convexity of Wright Functions with respect to Certain Functions}

The work in this section is motivated by the works of Baricz, Orhan and Yagmur, and Ponnusamy and Vuorinen [13, 15, 22, 23]. In this section we will discuss some conditions on the parameters $\lambda$ and $\mu$ under which the Wright functions are assured close-to-convex with respect to the functions

$$
\begin{gathered}
-\log (1-z), \\
\frac{1}{2} \log \left(\frac{1+z}{1-z}\right) .
\end{gathered}
$$

By using Lemma 2, we will get the following results.

Theorem 12. If $\mu \geq 1$ and $\lambda \geq 1 / 2$, then $z \rightarrow \mathscr{W}_{\lambda, \mu}(z)$ is close-to-convex with respect to the function $-\log (1-z)$.

Proof. Set

$$
f(z)=\mathscr{W}_{\lambda, \mu}(z)=z+\sum_{n=2}^{\infty} b_{n-1} z^{n} .
$$

We have $b_{n-1}>0$ for all $n \geq 2$ and $b_{1} \leq \Gamma(\mu) / \Gamma(1+\mu) \leq 1$, by using the inequality $\Gamma(\lambda n+\mu) \geq \Gamma(n+\mu)$. To prove that $f(z)$ is close-to-convex with respect to the function $-\log (1-z)$, we use Lemma 2. Therefore, we have to prove that $\left\{n b_{n-1}\right\}_{n \geq 2}$ is a decreasing sequence. By a short computation, we obtain

$$
\begin{aligned}
& n b_{n-1}-(n+1) b_{n} \\
& =\frac{\Gamma(\mu)}{(n-1) !}\left[\frac{n}{\Gamma(\lambda(n-1)+\mu)}-\frac{n+1}{n \Gamma(\lambda n+\mu)}\right] \\
& =\frac{\Gamma(\mu)}{(n-1) !}\left[\frac{n^{2} \Gamma(\lambda n+\mu)-(n+1) \Gamma(\lambda(n-1)+\mu)}{n \Gamma(\lambda n+\mu) \Gamma(\lambda(n-1)+\mu)}\right] \\
& >0 .
\end{aligned}
$$

By using the conditions on parameters, we can easily observe that $n b_{n-1}-(n+1) b_{n}>0$ for all $n \geq 2$, and thus $\left\{n b_{n-1}\right\}_{n \geq 2}$ is a decreasing sequence. By Lemma 2 , it follows that $f(z)$ is close-to-convex with respect to the function $-\log (1-z)$.

Theorem 13. If $\mu \geq 1$ and $\lambda \geq 1$, then $z \rightarrow z \mathscr{W}{ }_{\lambda, \mu}\left(z^{2}\right)$ is closeto-convex with respect to the function $(1 / 2) \log ((1+z) /(1-z))$.

Proof. Set

$$
f(z)=z \mathscr{W}_{\lambda, \mu}\left(z^{2}\right)=z+\sum_{n=2}^{\infty} B_{2 n-1} z^{2 n-1} .
$$

Here $B_{2 n-1}=b_{n-1}=\Gamma(\mu) /(n-1) ! \Gamma(\lambda(n-1)+\mu)$, and therefore we have $b_{1}=\Gamma(\mu) / \Gamma(\lambda+\mu) \leq 1$ and $B_{2 n-1}>0$ for all $n \geq 2$.
To prove our main result we will prove that $\left\{(2 n-1) b_{n-1}\right\}_{n \geq 2}$ is a decreasing sequence. By a short computation, we obtain

$$
\begin{aligned}
& (2 n-1) b_{n-1}-(2 n+1) b_{n} \\
& =\frac{\Gamma(\mu)}{(n-1) !}\left[\frac{2 n-1}{\Gamma(\lambda(n-1)+\mu)}-\frac{2 n+1}{n \Gamma(\lambda n+\mu)}\right], \\
& =\frac{\Gamma(\mu)}{(n-1) !}\left[\frac{\left(2 n^{2}-n\right) \Gamma(\lambda n+\mu)-(2 n+1) \Gamma(\lambda(n-1)+\mu)}{n \Gamma(\lambda n+\mu) \Gamma(\lambda(n-1)+\mu)}\right]
\end{aligned}
$$

$>0$.

By using the conditions on parameters, we can easily observe that $(2 n-1) b_{n-1}-(2 n+1) b_{n}>0$ for all $n \geq 2$, and thus $\left\{(2 n-1) b_{n-1}\right\}_{n>2}$ is a decreasing sequence. By Lemma 2 it follows that $f(z)$ is close-to-convex with respect to the function $(1 / 2) \log ((1+z) /(1-z))$.

\section{Hardy Spaces of Wright Functions}

Hardy spaces of hypergeometric functions are recently studied by Ponnusamy [24]. Baricz [4] used the idea of Ponnusamy and found the Hardy spaces of Bessel functions. Yagmur and Orhan [25] studied the same problem for generalized Struve functions. Similarly, Yagmur [26] studied the problem for Lommel functions. For Hardy spaces related to some classes of analytic functions, we refer to [27-30].

Theorem 14. Let, $\alpha \in[0,1), \mu>((4-\alpha)+$ $\left.\sqrt{5 \alpha^{2}-28 \alpha+32}\right) / 2(1-\alpha)$. Then

(i) $\mathscr{W}_{\lambda, \mu} \in H^{1 / 1-2 \alpha}$ for $\alpha \in[0,1 / 2)$.

(ii) $\mathscr{W}_{\lambda, \mu} \in H^{\infty}$ for $\alpha \geq 1 / 2$.

Proof. From the definition of Hypergeometric function, we have

$$
{ }_{2} F_{1}(a, b, c ; z)=\sum_{n=0}^{\infty} \frac{(a)_{n}(b)_{n}}{(c)_{n}} \frac{z^{n}}{n !} .
$$

Now, we have

$$
\begin{aligned}
k+\frac{d z}{\left(1-z e^{i \gamma}\right)^{1-2 \alpha}} & =k+d z_{2} F_{1}\left(1,1-2 \alpha, 1 ; z e^{i \gamma}\right) \\
& =k+d \sum_{n=0}^{\infty} \frac{(1-2 \alpha)_{n}}{n !} e^{i \gamma n} z^{n+1},
\end{aligned}
$$

where $k, d \in \mathbb{C}, \alpha \neq 1 / 2$, and $\gamma$ is any real number. Also, we have

$$
\begin{aligned}
k+d \log \left(1-z e^{i \gamma}\right) & =k-d z_{2} F_{1}\left(1,1, z ; z e^{i \gamma}\right) \\
& =k-d \sum_{n=0}^{\infty} \frac{1}{n+1} e^{i \gamma n} z^{n+1} .
\end{aligned}
$$

This implies that $\mathscr{W}_{\lambda, \mu}$ is not of the forms $k+d z\left(1-z^{i \gamma}\right)^{2 \alpha-1}$ for $\alpha \neq 1 / 2$ and $k+d \log \left(1-z e^{i \gamma}\right)$ for $\alpha=1 / 2$, respectively. Also from Theorem 6(ii) $\mathscr{W}_{\lambda, \mu}$ is convex of order $\alpha$. Hence, by using Lemma 5, we have the required result. 
Theorem 15. Let $\lambda \geq 1, \mu>(1+\sqrt{5}) / 2$, and $f \in \mathscr{R}$, and then the convolution $\mathscr{W}_{\lambda, \mu} * f$ is in $H^{\infty} \cap \mathbb{R}$.

Proof. Let $h(z)=\mathscr{W}_{\lambda, \mu}(z) * f(z)$. Then it is clear that $h^{\prime}(z)=\mathscr{W}_{\lambda, \mu}(z) / z * f^{\prime}(z)$. Using Corollary 7(iv), we have $\mathscr{W}_{\lambda, \mu}(z) / z \in \mathscr{P}$. Since $f \in \mathscr{R}$, therefore by using Lemma 3 , we have $h \in \mathscr{R}$. It is also clear that $\mathscr{W}_{\lambda, \mu}(z) / z$ is an entire function and therefore $h$ is entire. This implies that $h$ is bounded. Hence, we have the required result.

Theorem 16. Let $\lambda, \mu \in \mathbb{R}$ with $\lambda \geq 1, \mu>(1+\sqrt{5-4 \alpha}) / 2(1-$ $\alpha), \alpha \in[0,1)$, and $z \in \mathcal{U}$. If $f \in \mathscr{R}(\beta)$, then $\mathscr{W}_{\lambda, \mu} * f \in \mathscr{R}(\gamma)$, where $\gamma=1-2(1-\alpha)(1-\beta)$.

Proof. Let $h(z)=\mathscr{W}_{\lambda, \mu}(z) * f(z)$. Then, $h^{\prime}(z)=\mathscr{W}_{\lambda, \mu}(z) / z *$ $f^{\prime}(z)$. Now from Theorem 6(iv), we have $\mathscr{W}_{\lambda, \mu}(z) / z \in \mathscr{P}(\alpha)$. By using Lemma 4 and the fact that $f^{\prime} \in \mathscr{P}(\beta)$, we have $h^{\prime}(z) \in P(\gamma)$, where $\gamma=1-2(1-\alpha)(1-\beta)$. Consequently, we have $h \in \mathscr{R}(\gamma)$.

Corollary 17. Let $0 \leq \alpha<1, \lambda \geq 1$, and $\mu>(1+$ $\sqrt{5-4 \alpha}) / 2(1-\alpha)$. If $f \in \mathscr{R}(\beta), \beta=(1-2 \alpha)(2-2 \alpha)$, then $\mathscr{W}_{\lambda, \mu}(z) * f(z) \in \mathscr{R}(0)$.

Corollary 18. Let $\lambda \geq 1$ and $\mu>(1+\sqrt{5}) / 2$. If $f \in \mathscr{R}(1 / 2)$, then $\mathscr{W}_{\lambda, \mu}(z) * f(z) \in \mathscr{R}(0)$.

\section{Particular Case}

At the end of this paper, we give some particular cases of the above-mentioned theorem. When we put $\lambda=1$ and $\mu=5 / 2$ in (16), we obtain the function

$$
\mathscr{W}_{1,5 / 2}(-z)=\frac{3}{4}\left(\frac{\sin (2 \sqrt{z})}{2 \sqrt{z}}-\cos (2 \sqrt{z})\right) .
$$

By using Theorem 6 assertions (i) and (iv), we get the following corollary.

Corollary 19. (i) If $0 \leq \alpha<\alpha_{0}$, where $\alpha_{0} \simeq 0.0332 \cdots$, then $\mathscr{W}_{1,5 / 2} \in \mathcal{S}^{*}(\alpha)$. $\mathscr{P}(\alpha)$.

(ii) If $0 \leq \alpha<\alpha_{1}$, where $\alpha_{1} \simeq 0.4310 \cdots$, then $\mathscr{W}_{1,5 / 2} / z \in$

\section{Competing Interests}

The authors declare that there is no conflict of interests regarding the publication of this paper.

\section{Acknowledgments}

The research of Sarfraz Nawaz Malik is supported by COMSATS Institute of Information Technology, Pakistan, Reference no. 3-64/IPF-SRG/CIIT/Wah/14/765.

\section{References}

[1] P. L. Duren, Theory of $\mathrm{H}^{p}$ Spaces, vol. 38 of $A$ series of Monographs and Textbooks in Pure and Applied Mathematics, Academic Press, New York, NY, USA, 1970.
[2] O. Altintaş, O. Özkan, and H. M. Srivastava, "Neighborhoods of a class of analytic functions with negative coefficients," Applied Mathematics Letters, vol. 13, no. 3, pp. 63-67, 2000.

[3] H. Silverman, "Univalent functions with negative coefficients," Proceedings of the American Mathematical Society, vol. 51, pp. 109-116, 1975.

[4] Á. Baricz, "Bessel transforms and Hardy space of generalized Bessel functions," Mathematica, vol. 48, no. 2, pp. 127-136, 2006.

[5] J. K. Prajapat, "Certain geometric properties of the Wright function," Integral Transforms and Special Functions, vol. 26, no. 3, pp. 203-212, 2015.

[6] E. M. Wright, "On the coefficients of power series having exponential singularities," Journal London Mathematical Society, vol. 8, pp. 71-79, 1933.

[7] I. Podlubny, Fractional Differential Equations, Academic Press, San Diego, Calif, USA, 1999.

[8] S. G. Samko, A. A. Kilbas, and O. I. Marichev, Fractional Integrals and Derivatives: Theory and Applications, Gordon and Breach, New York, NY, USA, 1993.

[9] R. Gorenflo, Y. Luchko, and F. Mainardi, "Analytical properties and applications of the Wright function," Fractional Calculus \& Applied Analysis, vol. 2, no. 4, pp. 383-414, 1999.

[10] S. S. Miller and P. T. Mocanu, "Univalence of Gaussian and confluent hypergeometric functions," Proceedings of the American Mathematical Society, vol. 110, no. 2, pp. 333-342, 1990.

[11] S. Ruscheweyh and V. Singh, "On the order of starlikeness of hypergeometric functions," Journal of Mathematical Analysis and Applications, vol. 113, no. 1, pp. 1-11, 1986.

[12] Á. Baricz, Generalized Bessel Functions of the First Kind, vol. 1994 of Lecture Notes in Mathematics, Springer, Berlin, Germany, 2010.

[13] A. Baricz, "Geometric properties of generalized Bessel functions," Publicationes Mathematicae Debrecen, vol. 73, no. 1-2, pp. 155-178, 2008.

[14] Á. Baricz and S. Ponnusamy, "Starlikeness and convexity of generalized Bessel functions," Integral Transforms and Special Functions. An International Journal, vol. 21, no. 9-10, pp. 641653, 2010.

[15] H. Orhan and N. Yagmur, "Geometric properties of generalized Struve functions," Annals of the Alexandru Ioan Cuza University-Mathematics, 2014.

[16] N. Yağmur and H. Orhan, "Partial sums of generalized Struve functions," Miskolc Mathematical Notes, vol. 17, no. 1, pp. 657670, 2016.

[17] S. Owa, M. Nunokawa, H. Saitoh, and H. M. Srivastava, "Closeto-convexity, starlikeness, and convexity of certain analytic functions," Applied Mathematics Letters, vol. 15, no. 1, pp. 63-69, 2002.

[18] S. Ozaki, "On the theory of multivalent functions," Science Reports of the Tokyo Bunrika Daigaku, vol. 2, pp. 167-188, 1935.

[19] J. Stankiewicz and Z. Stankiewicz, "Some applications of Hadamard convolutions in the theory of functions," Annales Universitatis Mariae Curie-Sklodowska, vol. 40, pp. 251-265, 1986.

[20] S. Ponnusamy, "Inclusion theorems for convolution product of second order polylogarithms and functions with the derivative in a halfplane," Rocky Mountain Journal of Mathematics, vol. 28, no. 2, pp. 695-733, 1998.

[21] P. J. Eenigenburg and F. R. Keogh, "The Hardy class of some univalent functions and their derivatives," The Michigan Mathematical Journal, vol. 17, pp. 335-346, 1970. 
[22] S. Ponnusamy and M. Vuorinen, "Univalence and convexity properties for confluent hypergeometric functions," Complex Variables, Theory and Application, vol. 36, no. 1, pp. 73-97, 1998.

[23] S. Ponnusamy and M. Vuorinen, "Univalence and convexity properties for Gaussian hypergeometric functions," Rocky Mountain Journal of Mathematics, vol. 31, no. 1, pp. 327-353, 2001.

[24] S. Ponnusamy, "The Hardy spaces of hypergeometric functions," Complex Variables, Theory and Application, vol. 29, no. 1, pp. 8396, 1996.

[25] N. Yagmur and H. Orhan, "Hardy space of generalized Struve functions," Complex Variables and Elliptic Equations, vol. 59, no. 7, pp. 929-936, 2014.

[26] N. Yagmur, "Hardy space of Lommel functions," Bulletin of the Korean Mathematical Society, vol. 52, no. 3, pp. 1035-1046, 2015.

[27] J. H. Choi, Y. C. Kim, and H. M. Srivastava, "Convex and starlike generalized hypergeometric functions associated with the Hardy spaces," Complex Variables, vol. 31, pp. 345-355, 1996.

[28] Y. C. Kim and H. M. Srivastava, "The Hardy space for a certain subclass of Bazilevič functions," Applied Mathematics and Computation, vol. 183, no. 2, pp. 1201-1207, 2006.

[29] Y. C. Kim, K. S. Lee, and H. M. Srivastava, "Certain classes of integral operators associated with the Hardy space of analytic functions," Complex Variables, Theory and Application, vol. 20, no. 1-4, pp. 1-12, 1992.

[30] G. Miclãus, "The Libera generalized integral operator and Hardy spaces," Mathematical Notes. A Publication of the University of Miskolc, vol. 4, no. 1, pp. 39-43, 2003. 


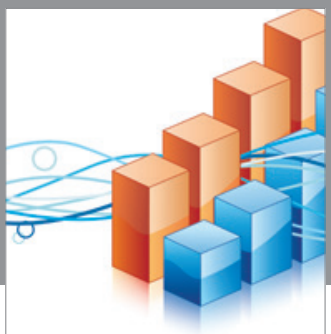

Advances in

Operations Research

vatem alat4

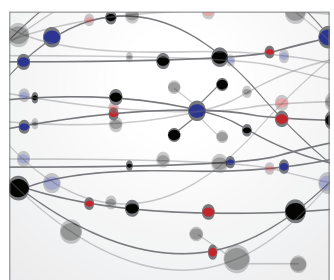

\section{The Scientific} World Journal
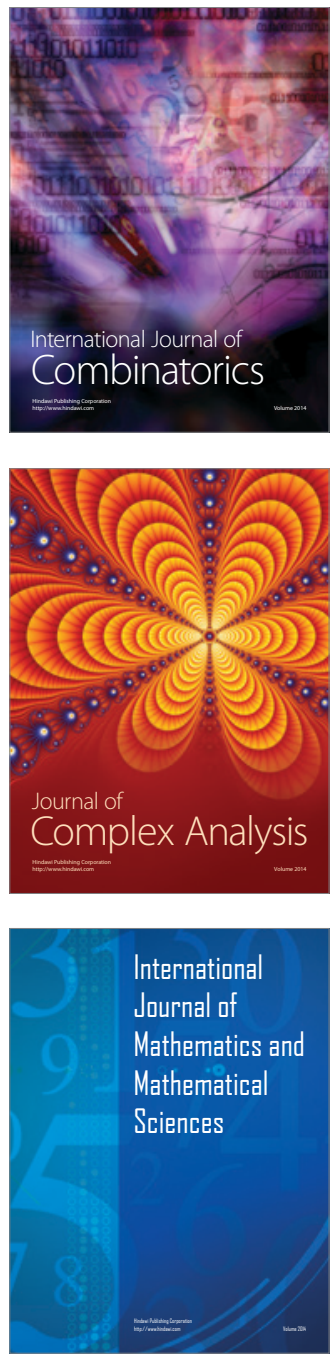
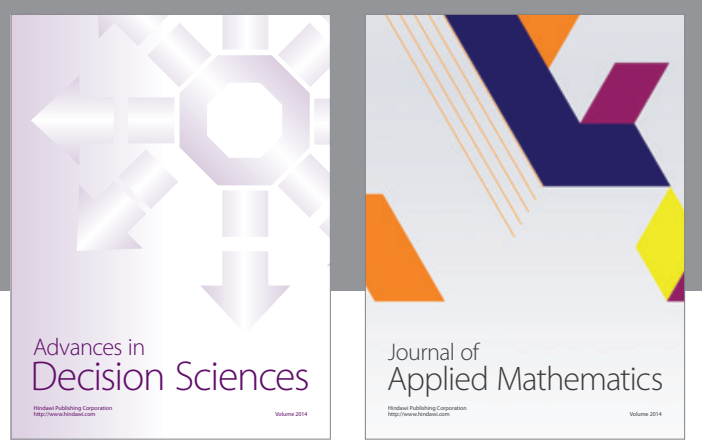

Algebra

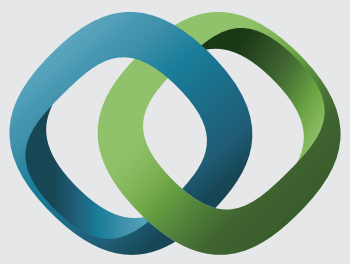

\section{Hindawi}

Submit your manuscripts at

http://www.hindawi.com
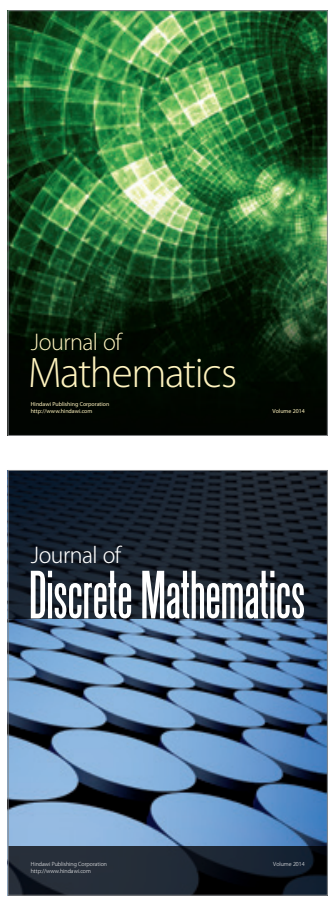

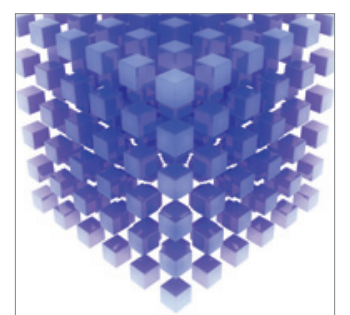

Mathematical Problems in Engineering
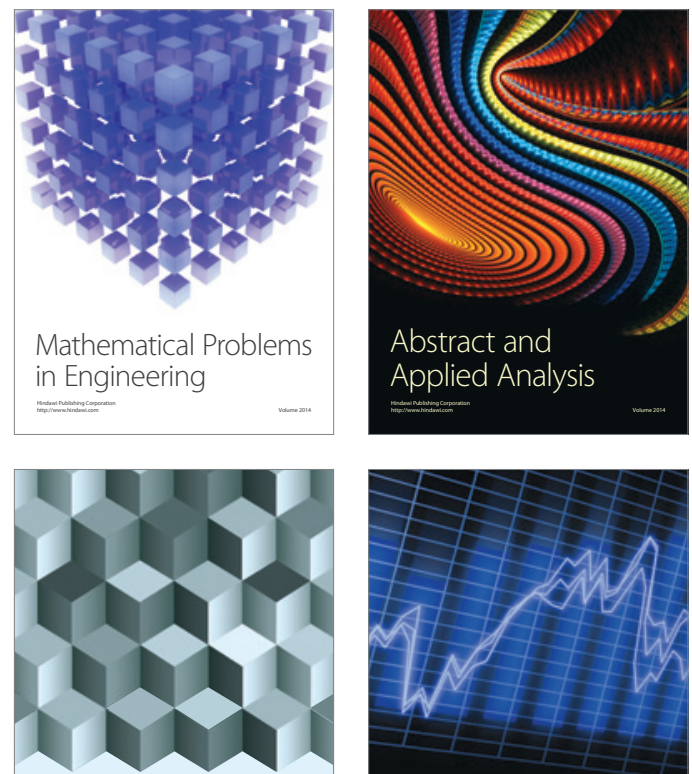

Journal of

Function Spaces

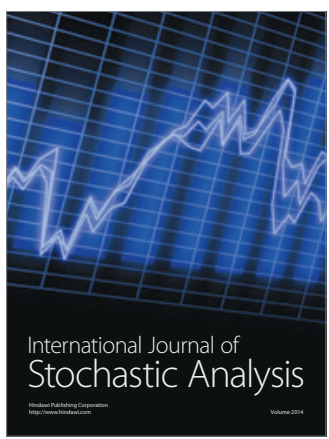

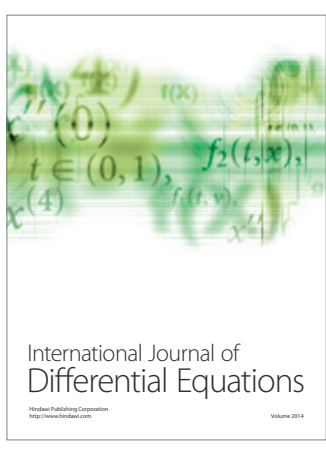
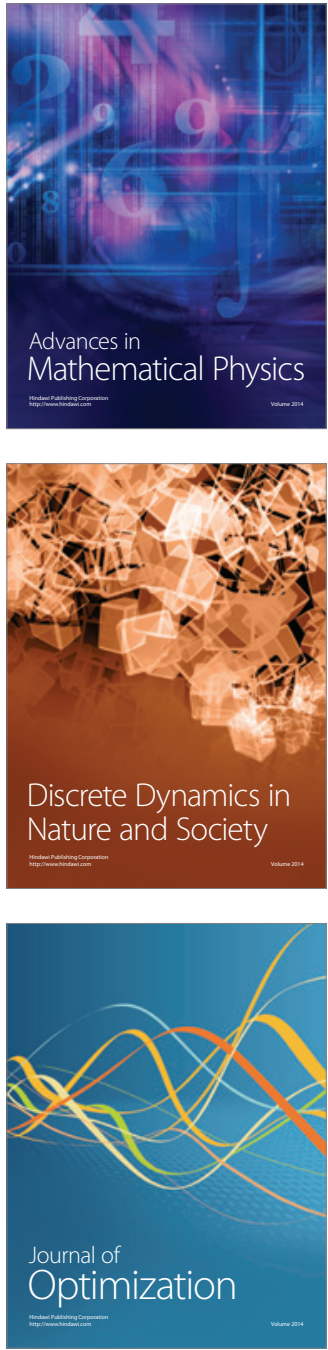